Check for updates

Cite this: Mater. Adv., 2021, 2, 3107

Received 1st March 2021, Accepted 29th March 2021

DOI: 10.1039/d1ma00175b

rsc.li/materials-advances

\title{
Graphene oxide-derived carbonaceous sensor: turn-off fluorescence sensor for nanomolar detection of ruthenium ions in aqueous medium $\dagger$
}

\author{
Jeyaraj Belinda Asha, ${ }^{\mathrm{a}}$ Murugan Karthik ${ }^{\mathrm{ab}}$ and Palaniswamy Suresh (D) *a
}

\begin{abstract}
Ruthenium is a vital transition metal widely employed in diverse applications from medicinal to material applications. Though ruthenium has been positioned as safe and relatively less toxic than other metals, continuous exposure will cause undesirable effects. Due to the constantly increasing usage of ruthenium, a simple and straightforward detection methodology is immediately necessary. In the present study, a covalently functionalized graphene oxide derived chemosensor has been developed to detect $\mathrm{Ru}(\mathrm{II})$ under a complete aqueous medium. For this, graphene oxide (GO) is covalently functionalized on the edge carboxylic acid group with 2-(bis(pyridine-2-ylmethyl)amino)ethan-1-ol (PAE) through esterification, followed by further grafting on the basal plane with a dansyl fluorophore motif through a silyloxy propyl linkage. Each functionalization has been designed to create a feasible pathway for a Förster Resonance Energy Transfer (FRET) donor and acceptor energy transfer, where the metal-binding site is introduced through esterification and the signalling unit by the dansylation process. When exploring the application of the newly prepared GOPND carbonaceous material, a selective fluorescence quenching response has been observed with $\mathrm{Ru}(\mathrm{III})$ in an aqueous medium, which is utilized for the detection of Ru(III). A GOPND probe showed high selectivity towards Ru(II) over the presence of other common metal ions with a lower detection limit of $15 \mathrm{nM}$. The origin of this 'turn-off' sensing response is explained through the presence of both the bis(pyridyl) probe and the dansyl fluorophore in close proximity on $\mathrm{GO}$, which facilitates the energy transfer. The specific turn-off fluorescence response of the GOPND with Ru(III) ions is rationalized through the FRET mechanism.
\end{abstract}

\section{Introduction}

Graphene oxide (GO) is a unique and versatile carbonaceous material in the contemporary materials research field. ${ }^{1-6}$ GO has attracted massive attention in developing novel materials for real-time applications due to its inherent and exceptional physical, $^{7}$ chemical, $^{8}$ electronic, and optical properties. ${ }^{9}$ Though graphene and graphene oxide (GO) have growing demand in material-based applications, ${ }^{10}$ the most exciting aspects of the graphene research field are developing

\footnotetext{
${ }^{a}$ Supramolecular and Catalysis Lab, Department of Natural Products Chemistry, School of Chemistry, Madurai Kamaraj University, Madurai - 625021, India. E-mail: suresh.chem@mkuniversity.ac.in, ghemistry@gmail.com; Tel: +919790296673

${ }^{b}$ Department of Chemistry, National Institute of Technology, Tiruchirappalli, India $\dagger$ Electronic supplementary information (ESI) available: Fig. S1 and S2: ${ }^{1} \mathrm{H}$ and ${ }^{13} \mathrm{C}$ NMR of PAE; Fig. S3: ESI-MS; Fig. S4: EDX mapping; Fig. S5 and S6: UV-vis and emission studies; Fig. S7: UV-vis spectral studies of all metal analytes; Fig. S8: PL spectral studies at different wavelengths; Fig. S9: PL studies of all metal analytes; Fig. S10 and S11: Control experiments with PL studies; Fig. S12: FTIR; Fig. S13: Stern-Volmer plot. See DOI: 10.1039/d1ma00175b
}

functionalized graphene materials with organic and inorganic motifs to enhance or fine-tune their functional properties. ${ }^{11}$ Due to the remarkable optical and electronic properties of covalently modified $\mathrm{GO},{ }^{12}$ it has received enormous attention in optical and sensor applications. ${ }^{13}$ Though several physisorbed sensor probes have been reported in the literature, poor signal reversibility and reproducibility, leaching of the physisorbed probes, and loss of active sites have hindered the development of a reliable sensor system. ${ }^{14}$ False-positive signals were detected in many cases of physisorbed or non-covalent functionalized GO sensors, which strongly insist on the need for covalent functionalization. ${ }^{14,15}$ Notably, interaction with other molecules or ions, and optical and luminescent behaviours have been significantly improved through covalent functionalization. ${ }^{16}$ The intrinsic electronic property of GO yields a special characteristic feature of the energy acceptor in the energy transfer process. ${ }^{17,18}$ In GO-based sensors, the fluorescence resonance energy transfer process takes place predominantly, and GO acts as an acceptor for the efficient quenching of nearby fluorescence or through the covalently attached species owing to the $\mathrm{sp}^{2}$ domains. $^{19}$ 
Though the photophysical nature of GO has been widely studied as a quencher, under certain environments and in the presence of other external stimuli, the luminescent nature of GO was enhanced. Such an increase in the PL behavior of GO and its physisorbed or chemically modified derivatives have been used for diverse sensing applications. ${ }^{20-23}$ Recently, the chemosensor application of GO-derived materials has been continuously explored in the detection of heavy metal ions ${ }^{24}$ and even for $f$-block elements, ${ }^{25}$ organophosphorus pesticides, ${ }^{26}$ and ATP and GTP monitoring in living cells. ${ }^{27} \mathrm{~A}$ maximum number of reports have focused on the quenching of the PL through either blocking or unblocking of the graphitic surface of GO through physisorbed molecules over the surface ${ }^{28}$ or decorated nanoparticles. ${ }^{29}$ Recently, the detection $^{30,31}$ and removal of chromium(IV) ${ }^{32}$ ions was proposed using a graphenebased system. In GO-based sensors, the non-covalently modified system has shown excellent response; but, indeed, none of the studies disclosed their stability or reproducibility for further applications, which strongly questions the reliability of such sensor materials. There is a lack of adequate studies on the stability of non-covalently modified GO-based sensors. With this ambiguity, in the present work, we have designed and developed a turn-off fluorescence sensor through covalent modification of both the edge carboxyl group and the basal plane of GO. A turn-off sensor material has been designed and developed based on the fluorescence resonance energy transfer between the coordinated metal site and a luminescent probe attached on GO, where an organic motif 2-bis(pyridine-2-ylmethyl)amino)ethan-1ol (PAE) was used as a metal coordinating site, which was covalently attached to GO through Steglich ${ }^{33}$ esterification and a dansyl motif as a fluorescence probe was grafted on the basal plane through a propyl silyloxy linkage. This sensing material is designed to enhance benignity and also to detect ruthenium metal in an aqueous medium. With such a perspective, GOPND has been designed with a FERT-based sensing mechanism.

Ruthenium is a rare and heavy transition metal which has diverse attractive applications from medicine to material applications. Ruthenium has been found to be a potential alternative to platinum in medicinal applications and is used as an immunosuppressant ${ }^{34}$ and dental alloy, ${ }^{35}$ as an antiAlzheimer's agent, ${ }^{36}$ in microbial antibiotic production for Malaria, Chagas disease, septic shocks, and cancer treatment. ${ }^{34}$ Ruthenium-derived drugs are gaining more attention in clinical application owing to their low toxicity. ${ }^{34,36}$ Meantime, ruthenium is an inevitable candidate in electronics due to its excellent bandgap energies; it has been employed in semiconductor and thermoelectric applications. ${ }^{37}$ In contemporary research on energy harvesting from renewable resources, notably from sunlight, the dye-sensitized solar cell has played a significant role. ${ }^{38}$ The redox properties of ruthenium are effectively utilized to enhance the efficiency of light energy harvesting. ${ }^{38,39}$ On the other hand, ruthenium plays an inevitable role in synthetic organic chemistry as a potential catalyst. After the 2005 Noble prize was awarded for olefine metathesis to form a C-C bond, ${ }^{40-42}$ the demand for ruthenium-derived catalysts has increased exponentially for synthesizing active pharmaceutical ingredients.
Besides, reactions such as Fischer-Tropsch ${ }^{43}$ and BoultonKatritzky $^{44}$ also utilize ruthenium as a catalyst. In asymmetric synthesis, ruthenium-derived chiral catalysts are employed to fine-tune the enantioselectivity. ${ }^{45}$ However, it is challenging to remove and quantify the deeply colored ruthenium by-products from the desired product. ${ }^{46}$ Though ruthenium has found numerous applications and plays a pivotal role in human welfare, its fate after usage has not been determined correctly. Indeed, exposure to, extensive use of, and improper disposal of ruthenium ions could also cause adverse effects in the environment, because they exhibit various forms of toxicity and lead to health issues like the platinum group elements (PGEs). ${ }^{47-51}$ The maximum permissible intake of $\mathrm{Ru}(\mathrm{III})$ or related transition metals in oral dosage and parenteral dosage in pharmaceutical products per day are $10 \mu \mathrm{g} \mathrm{g}^{-1}$ and $1.0 \mu \mathrm{g} \mathrm{g}^{-1}$ respectively. ${ }^{47-53}$ Apart from its extensive applications, in general, most ruthenium-containing compounds are considered to be toxic because they can deeply stain the skin, and the ingested metal may be retained strongly in the bones. ${ }^{34}$ Excess exposure to or intake of ruthenium is harmful and may cause corrosion to the respiratory tract, eyes, and digestive tract. ${ }^{54}$ The toxic nature of ruthenium is also taken quite seriously since it has a mutagenic effect in normal cells. ${ }^{54-56} \mathrm{~A}$ more extended stay of a ruthenium complex in the lungs results in metastatic tumours. ${ }^{54,55}$ To detect the existence of ruthenium ions, routine analytical techniques such as atomic absorption $(\mathrm{AAS})^{57}$ and inductively coupled plasma-mass spectrometry (ICP-MS) are used. ${ }^{58}$ However, they involve expensive instrumental analysis and a rigorous sample preparation method. To the best of our knowledge, there has been no study on the development of a chemosensor for the detection of ruthenium except for a few reports. A colorimetric methodology was reported using per-6amino- $\beta$-cyclodextrin as a supramolecular host to detect $\mathrm{Ru}(\mathrm{III}) .{ }^{59}$ A few fluorescence detectors, such as porphyrin macrocyclics, ${ }^{60}$ fluorescein derivatives through the oxidative process, ${ }^{61} \mathrm{CdTe}$ quantum dots ${ }^{62}$ and a 3D luminescent indium-organic framework $^{63}$ and functionalized phenanthridine derivatives, have been used to detect $\mathrm{Ru}(\mathrm{III})^{64}$ and an aggregation-induced emission-based probe for $\mathrm{Ru}(\mathrm{III})$ has also been reported. ${ }^{65}$ Though numerous metal detecting methodologies and chemosensors have continuously evolved for several common metal ions, very few sensing methods have been developed for the biologically and industrially essential metal ruthenium. Indeed, a suitable and selective detection methodology is an immediate need. A reliable and straightforward sensing technique must be designed to detect such pharmaceutically cum industrially versatile metal ions from the environment and biological systems.

We have developed a "turn-off" fluorescence sensing strategy for the rapid and selective detection of ruthenium(III) in an aqueous medium. Based on hard acid and soft base (HSAB) theory, a bis(pyridine) motif as a metal coordination site, and dansyl motifs attached to GO as a fluorescent probe, a heterogeneous fluorescence sensing probe has been designed to operate through a FRET sensing mechanism. With this fundamental design, when ruthenium(III) coordinates with GOPND and under subsequent excitation, an excited state energy transfer takes place through FRET from ruthenium(III) 
coordinated bis(pyridyl) to dansyl attached on the basal plane of GO, and a simple turn-off type fluorescence sensing process takes place. The calculated LOD is also relatively lower than previously reported values. Furthermore, unlike homogenous chemosensors, heterogeneous chemosensors will strongly support scaling up and the design of materials for real-time sensor applications.

\section{Experimental section}

\section{Materials and methods}

2-Aminoethanol (Sd Fine-chem. Ltd), 2-(chloromethyl)pyridine hydrochloride, and 4-dimethylaminopyridine, $N, N^{\prime}$-dicyclohexylcarbodiimide (Spectrochem) were used as received. Pristine graphite (Sigma-Aldrich), $\mathrm{NaNO}_{3}$, potassium permanganate, sulfuric acid, hydrochloric acid, potassium carbonate, $\mathrm{AgNO}_{3}$, $\mathrm{Al}\left(\mathrm{NO}_{3}\right)_{3} \cdot 9 \mathrm{H}_{2} \mathrm{O}, \mathrm{BiCl}_{3}, \mathrm{CaCl}_{2}, \mathrm{CoCl}_{2}, \mathrm{CuSO}_{4} \cdot 5 \mathrm{H}_{2} \mathrm{O}, \mathrm{Cr}_{2}\left(\mathrm{SO}_{4}\right)_{3}$, $\mathrm{FeCl}_{3}, \mathrm{HgSO}_{4}, \mathrm{KCl}, \mathrm{La}_{2}\left(\mathrm{SO}_{4}\right)_{3}, \mathrm{Mg}\left(\mathrm{NO}_{3}\right)_{2}, \mathrm{NaCl}, \mathrm{Ni}\left(\mathrm{NO}_{3}\right)_{2} \cdot 6 \mathrm{H}_{2} \mathrm{O}$, $\mathrm{Pd}\left(\mathrm{NO}_{3}\right)_{2}, \mathrm{Rh}_{2}\left(\mathrm{CH}_{3} \mathrm{COO}\right)_{4}, \mathrm{RuCl}_{3}, \mathrm{SnCl}_{2}, \mathrm{ZnCl}_{2}$ and $\mathrm{ZrCl}_{4}$ (Merck), triethylamine, (3-aminopropyl)triethoxysilane, and dansyl chloride (Alfa Aesar) were used as received without further purification.

\section{Characterization}

Elemental composition (CHN) was examined using a PerkinElmer USA, 2400 series 2 instrument. Fourier-transform infrared (FTIR) spectra were documented with a Shimadzu instrument from $4000 \mathrm{~cm}^{-1}$ to $500 \mathrm{~cm}^{-1}$, using the $\mathrm{KBr}$ pellet technique. FT-Raman spectra were studied with a Shimadzu instrument with a $630 \mathrm{~nm}$ laser. Powder X-ray diffraction (PXRD) was analyzed (XPERT-PRO) using $\mathrm{Cu} \mathrm{K} \alpha$ radiation at room temperature. Scanning Electron Microscopy (SEM) analysis was performed with a TESCAN VEGA3 instrument using an SE detector and furnished with Energy Dispersive Analysis of X-rays (EDX). High-Resolution Transmission Electron Microscopy (HR-TEM) images of samples were obtained with a FEI-TECNAI G2 200 KV TEM instrument. $\mathrm{X}$-ray photoelectron spectroscopy (XPS) was recorded using an Omicron Nanotechnology spectrometer $(h \nu=1486.6 \mathrm{eV}) .{ }^{1} \mathrm{H}$ NMR and ${ }^{13} \mathrm{C}$ NMR spectra were analyzed using Bruker spectrometers of $300 \mathrm{MHz}$ and $75 \mathrm{MHz}$, respectively. Electrospray ionization mass spectrometry (ESI-MS) analysis was performed with a machine from LCQ Fleet-Thermo Fisher instruments Limited, USA. For the absorption and emission spectral studies, aqueous solutions of GOPND and all-metal solutions were prepared using double distilled water and the $\mathrm{pH}$ was maintained at 7 using PBS (phosphate-buffered saline) buffer. UV-vis spectra were recorded with an Agilent diode array 8453 spectrophotometer. Photoluminescence (PL) studies were conducted using a HoribaJobin-Yvon FluoroMax 3 instrument.

\section{Synthesis of PAE and GOP}

Synthesis of GOPND involves three different steps. Initially, PAE was synthesized using the simple coupling reaction procedure by adopting the method in previous literature ${ }^{66}$ and GO was prepared by the modified Hummers' method. ${ }^{67}$ Typical esterification was carried out with the Steglich protocol, ${ }^{33}$ using GO and PAE in which the edge carboxylic group of GO was functionalized as an ester using the hydroxyl group of PAE. Fig. S1-S3 (ESI $\dagger$ ) all represent the $\mathrm{H}^{1}, \mathrm{C}^{13}$ NMR and LC-MS of PAE. The prepared materials were systematically characterized.

\section{Synthesis of GOPN (functionalization of GOP with (3- aminopropyl)triethoxysilane)}

The as-prepared GOP $(100 \mathrm{mg})$ was taken in dry THF $(5 \mathrm{~mL})$ under sonication for 30 minutes for uniform dispersion; then (3-aminopropyl)triethoxysilane (APTES) ${ }^{68}(120 \mu \mathrm{L})$ was added to the dispersed GOP solution and allowed to reflux in an RB fitted with a water condenser for efficient cooling under stirring for $24 \mathrm{~h}$. After the solution had cooled to room temperature, the resulting material was separated by centrifugation and washed with $(3 \times 10 \mathrm{~mL})$ acetone three times. Then it was filtered through a nylon membrane and dried under vacuum in a glass oven. The obtained material was stable at room temperature and was characterized using various analytical techniques.

\section{Synthesis of GOPND (Dansylation of GOPN)}

GOPN (75 mg) was taken in dry THF (5 mL) and sonicated for 30 minutes to get a uniform dispersion. Dansyl chloride $(30 \mathrm{mg})$ dissolved in $5 \mathrm{~mL}$ of THF was added to the GOPN dispersion, followed by the addition of triethylamine $(500 \mu \mathrm{L}){ }^{69}$ and the sonication was continued for complete dispersion; then it was stirred at room temperature for about five hours. TLC used to periodically monitored the dansylation reaction. The total consumption of dansyl chloride was followed by the disappearance of the dansyl chloride spot in TLC. The resulting dansylated GOP material was separated by centrifugation, followed by three washings with acetone and filtering through a nylon membrane and drying under vacuum in a glass oven; the obtained material was systematically analyzed and characterized with the help of various analytical techniques.

\section{Results and discussion}

In the present work, a carbonaceous sensor was developed from graphene oxide (GO) through covalent functionalization with an energy donor and acceptor to detect $\mathrm{Ru}(\mathrm{III})$ using the fluorescence technique in an aqueous medium (Scheme 1). First, 2-bis(pyridine-2-ylmethyl)amino)ethylgraphenate (GOP) was prepared from GO through Steglich esterification with PAE. Then the graphitic plane of GOP was covalently grafted with silyloxy aminopropyl using APTES, and subsequently, the primary amino group present on the resulting GOPN was reacted with dansyl chloride, finally giving the probe GOPND. In GOPND, the bis(pyridyl) motif and the dansyl probe are covalently attached to the edge carboxylic group and on the basal plane of GO, respectively. This clearly explains that the preparation process is step-wise. The as-prepared GOPND sensor material and its corresponding precursors were subjected to systematic characterization using various spectroscopic and microscopic analyses at each stage of preparation. 

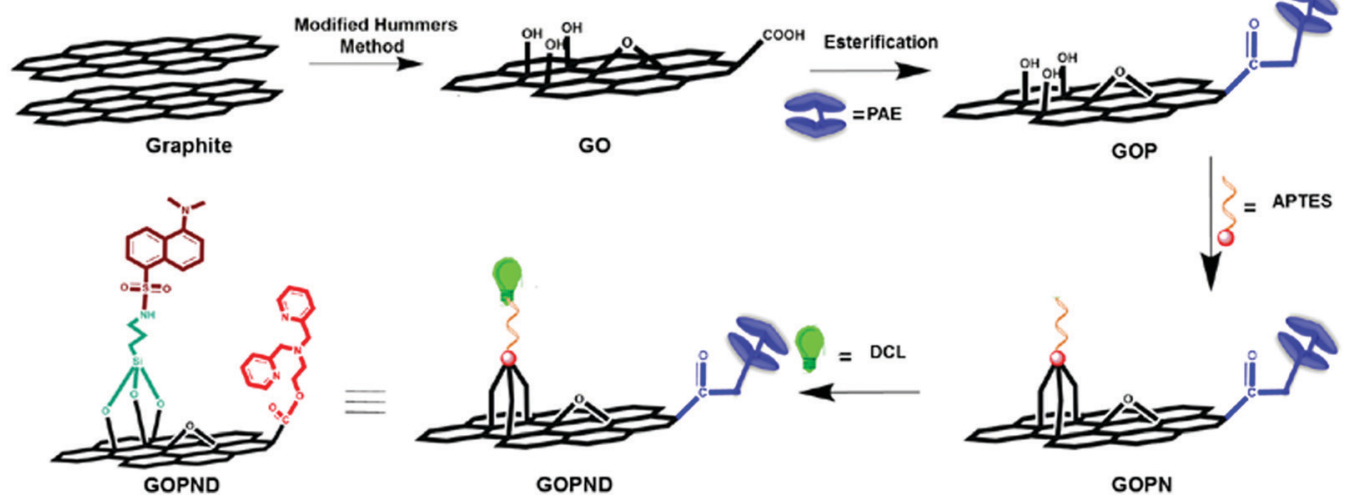

Scheme 1 Synthetic route for the preparation of GOPND.

All characterizations precisely revealed the unique functionalization. The preliminary elemental analysis results of GOP, GOPN, and GOPND showed a notable increase in the elemental composition (Fig. 1(i)). A significant enhancement in the elemental ratio and $12 \%$ nitrogen support the formation of GOPND.

Next, the covalent functionalization was characterized using Fourier transform infrared (FT-IR) spectroscopy analysis; this is an elegant tool to analyze the chemical functionalization of graphitic materials. The complete covalent modification and the formation of GOPND are confirmed through the meticulous analysis of the FT-IR spectra and a comparison with its precursors (Fig. 1(ii)). The presence of a peak at $1722 \mathrm{~cm}^{-1}$ represents the $\mathrm{C}=\mathrm{O}$ stretching of the ester linkage and indicates the functionalization of the bis(pyridyl) motif on the edge carboxylic groups of GO. Added to that, the stretching at $1575 \mathrm{~cm}^{-1}$ of the aromatic $\mathrm{C}=\mathrm{C}$ stretching shows the presence of the pyridyl rings of the bis(pyridyl) motif. Compared to GO, new peaks that appeared in the range of $1032 \mathrm{~cm}^{-1}$ to $1122 \mathrm{~cm}^{-1}$ corresponding to the $\mathrm{Si}-\mathrm{O}$ and $\mathrm{Si}-\mathrm{O}-\mathrm{C}$ stretching frequencies confirmed the functionalization of aminopropyl on the basal plane of GO through silyloxy linkage. Further, the peak at $3377 \mathrm{~cm}^{-1}$ of the primary amine in GOPN shifted to $3405 \mathrm{~cm}^{-1}$ in GOPND, corresponding to the stretching frequency of the secondary amine, demonstrating the functionalization of dansyl chloride. Further peaks at $1390 \mathrm{~cm}^{-1}$ of sulfonyl, $2491 \mathrm{~cm}^{-1}$ of $\mathrm{S}=\mathrm{O}$ and $\mathrm{N}-\mathrm{H}$ stretching frequencies, and $1473 \mathrm{~cm}^{-1}$ of the symmetric bending stretching frequency of the methyl group support the functionalization of dansyl motifs with GOPN. The peaks at $785 \mathrm{~cm}^{-1}$ and $1520 \mathrm{~cm}^{-1}$ of naphthalene stretching frequencies also strongly ascertain the presence of the dansyl motif in the covalently functionalized GOPND. The peaks $2669 \mathrm{~cm}^{-1}$ and $2928 \mathrm{~cm}^{-1}$ of alkyl stretching frequencies show the propyl linkage between the dansyl motif and the graphitic plane of GOPND. Furthermore, the peaks at $1645 \mathrm{~cm}^{-1}$ and $1210 \mathrm{~cm}^{-1}$ of aromatic $\mathrm{C}=\mathrm{C}$ and $\mathrm{C}-\mathrm{C}$ ascertain the presence of the graphitic plane.

FT-Raman provides unique information about the graphitic material, such as the degree of functionalization and defects derived from the functionalization. All the as-prepared materials, GOP, GOPN, and GOPND, were analyzed by FT-Raman, and the
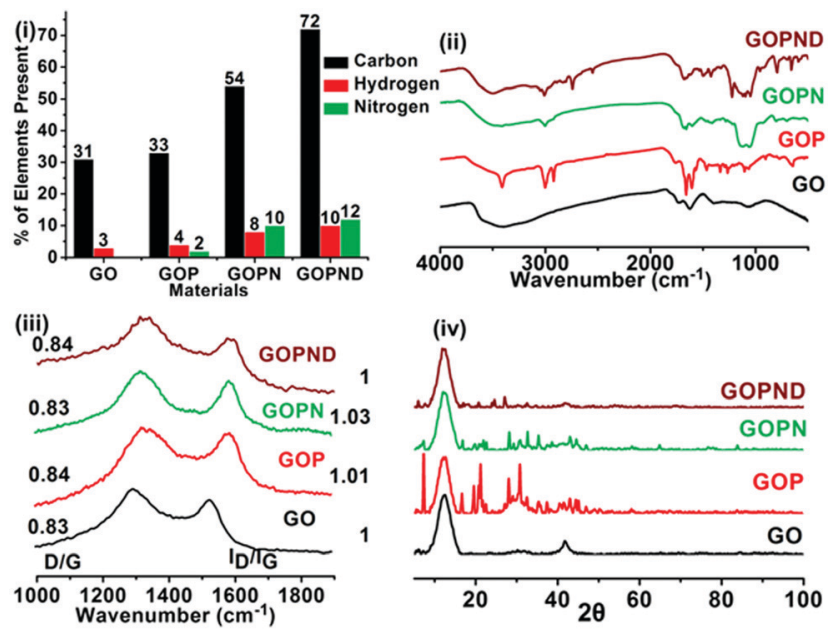

Fig. 1 Spectroscopic analysis of GO, GOP, GOPN, and GOPND, (i) elemental analysis, (ii) FTIR, (iii) Raman spectra, (iv) powder X-ray diffraction (P-XRD) pattern.

results are presented in Fig. 1(iii). From the Raman spectra, the existence of $\mathrm{sp}^{2}$ and $\mathrm{sp}^{3}$ hybridization and the corresponding change in the $\mathrm{D} / \mathrm{G}$ ratio of derivatized $\mathrm{GO}$ materials reveal functionalization. For GO and GOP, the calculated $\mathrm{D} / \mathrm{G}$ ratios are 0.83 and 0.84 , respectively, and $I_{\mathrm{D}} / I_{\mathrm{G}}$ are 1 and 1.01 , respectively, which indicates that no notable change occurs on the GO basal plane. Similar results continued on further functionalized GOPN and GOPND. The D/G ratios of GOPN and GOPND are 0.83 and 0.84 , respectively, and the $I_{\mathrm{D}} / I_{\mathrm{G}}$ ratios are 1.03 and 1.02 , respectively. The analysis of both $\mathrm{D} / \mathrm{G}$ and $I_{\mathrm{D}} / I_{\mathrm{G}}$ ratios revealed no significant changes apart from a marginal increase in the peak intensities. This discloses that the esterification and the silylation on the basal plane of GO did not affect the hybridization of the carbon on the graphitic domain because the silyloxy propyl functionalization takes place on the existing hydroxyl and epoxide functional groups. The FT-IR analysis also supports this observation through the unaffected $\mathrm{C}=\mathrm{C}$ peaks at $1626 \mathrm{~cm}^{-1}$.

Further, to verify the crystalline nature of the as-prepared GOP, GOPN, and GOPND, they were subjected to powder X-ray 
diffraction (PXRD) analysis. However, the PXRD pattern does not provide any remarkable information regarding the functionalization and formation of GOPND material. In general, GO shows two characteristic broad peaks at $2 \theta$ values of $12.2^{\circ}$ and $41.6^{\circ}$ corresponding to the $(001)^{70}$ and $(100)^{71}$ reflection planes, respectively. In the case of GOPND after covalent modification, the same two planes are retained, whereas the (001) planes are slightly shifted to $12.4^{\circ}$. However, compared to GO and GOP, the peaks get broader, which affirms that the amorphous nature has been maintained even after functionalization with silyloxy bonding. Indeed, the interlayer spacing of GO could be tuned through the covalent functionalization. The calculation of the interlayer spacing of GOPND using the Bragg's equation showed an intriguing enhancement in interlayer spacing of $2.3 \mathrm{~nm}$ from $0.725 \mathrm{~nm}$ with a slight decrease in $2 \theta(001)=$ 12.779 to 12.43 , which ascertained the covalent functionalization of GO and resulted from the formation of GOPND. Although all the above characterizations gave an understanding regarding the formation of GOPND from GO, finally, to unambiguously understand the covalent functionalization and confirm the formation of GOPND, X-photoelectron spectroscopy studies (XPS) were carried out (Fig. 2). When analyzing the XPS spectrum of GO, only two significant peaks of $\mathrm{C} 1 \mathrm{~s}(286.4 \mathrm{eV})$ and $\mathrm{O} 1 \mathrm{~s}$ $(533 \mathrm{eV})$ were present. After covalent modification, in the XPS spectrum of GOPND, in addition to the enhanced intensity of C 1s and $\mathrm{O} 1 \mathrm{~s}$, new additional peaks $\mathrm{N} \mathrm{1s}, \mathrm{S} 2 \mathrm{~s}$, and $2 \mathrm{P}$ and $\mathrm{Si} 2 \mathrm{p}$ appeared.

The deconvoluted C 1s spectrum at $284 \mathrm{eV}$ of GOPND gives three peaks at $283.6 \mathrm{eV}$ of $\mathrm{sp}^{2}$ of $\mathrm{C}-\mathrm{C}^{72}$ of the graphitic basal plane, at $284.7 \mathrm{eV}^{73}$ of the aromatic $\mathrm{C}-\mathrm{C}$ from benzene and naphthyl rings, and at $286.7 \mathrm{eV}$ of the $\mathrm{C}-\mathrm{O}$ from the ester carboxylate. $^{74}$ The peak at $531.3 \mathrm{eV}$ corresponds to $\mathrm{O} 1 \mathrm{~s}$, which validates the presence of ester carbonyl $(\mathrm{C}=\mathrm{O}) .{ }^{75}$ Next, deconvoluting the $\mathrm{N} 1 \mathrm{~s}$ peak at $399 \mathrm{eV}$ gives two peaks at $398.9 \mathrm{eV}$ and $401 \mathrm{eV}$ of the pyridinic nitrogen of the bis(pyridyl) unit and the ' $\mathrm{C}-\mathrm{N}$ ' of the $N, N$-dimethyl unit from the dansyl motif, respectively. These nitrogen peaks confirmed the covalent attachment of the bis(pyridyl) and dansyl groups to GO. Further, the presence of two peaks at $231 \mathrm{eV}$ and $167.6 \mathrm{eV}$ corresponds to the sulfur $2 \mathrm{~s}$ and $2 \mathrm{p}$ binding energy present as a sulfone group in the dansyl motif. Finally, the peak at $101.5 \mathrm{eV}$ proves the silyloxy functionalization on the GO basal plane and is further deconvoluted to give two peaks at $101.2 \mathrm{eV}$ of $\mathrm{Si}-\mathrm{O} /$ Si-O-C ${ }^{76}$ and $101.9 \mathrm{eV}$ of Si-C. ${ }^{77}$ The above-detailed observation from the XPS survey strongly confirms the formation of GOPND.

SEM images usually demonstrate a sheet-like morphology for GO; the same has been observed here, but in the case of GOP (Fig. 3(b)), certain voids can be detected due to the covalent modification. The SEM images show a crushed and pulverized form, which reveals that the functionalization may introduce a lot of defects over the surfaces. The sheets were not uniform; there are a lot of pits found on the surface due to its surface defects. Silylation introduces a flake-like morphology followed by a network-like morphology for fluorophoric modification. Fig. S4 of ESI $\dagger$ gives the elemental mapping of GOPND; also, the elemental percentage endorses the result of CHN analysis. HR-TEM images (Fig. 3e-i) show the same as SEM; the functionalized GO also formed in a sheet-like morphology with multiple stacking of layers in all the steps, and the presence of wrinkles on the sheets indicated their functionalization. The functionalized GOPND finally exists as a clear sheet-like morphology without any crumbling or flooding, which agrees with the SEM images.

From all the above spectroscopic and microscopic analyses, the covalent modification was unambiguously characterized and based on the characterization results a plausible structure of GOPND is proposed (Scheme 1).

After meticulously characterizing the as-prepared GOPND material, its application has been disclosed as a heterogeneous carbon-derived sensing probe to detect environmentally

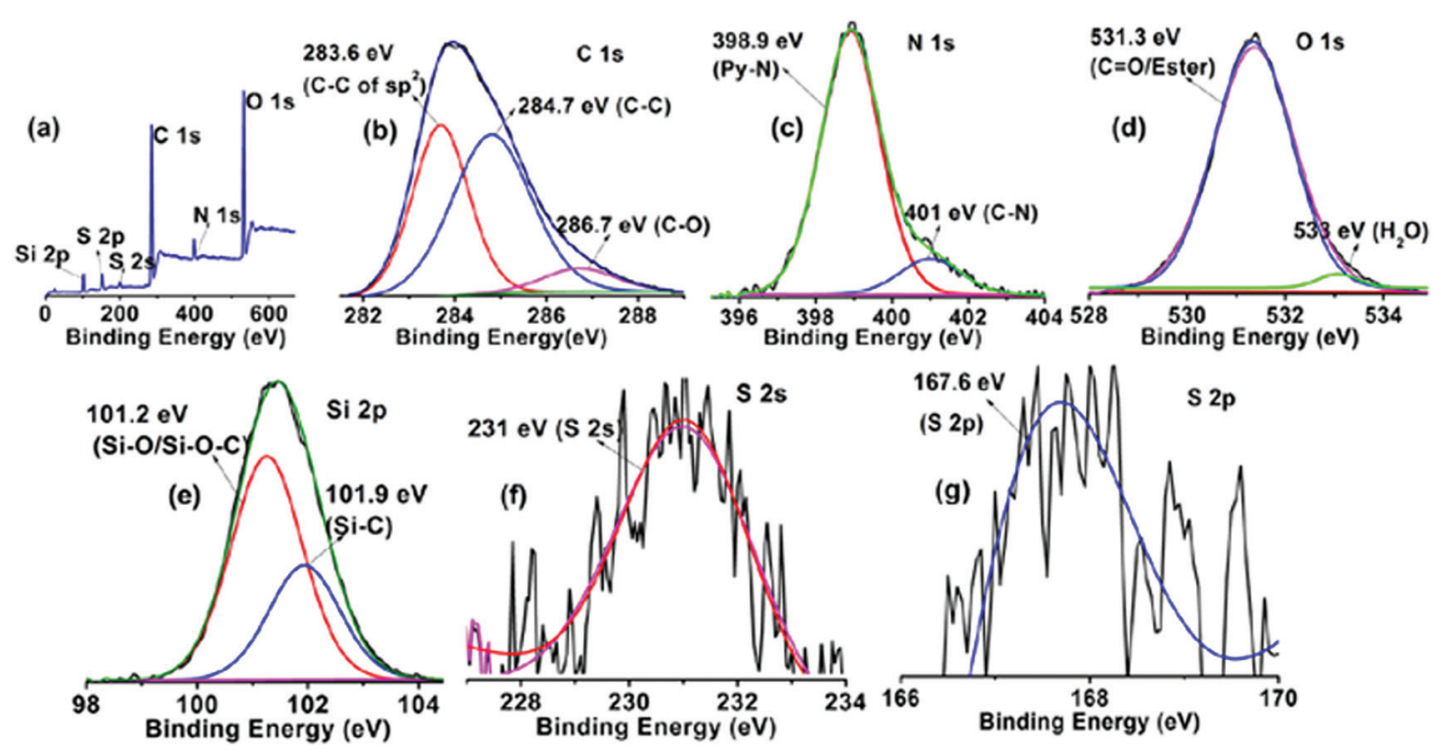

Fig. 2 XPS survey spectrum of GOPND (a). Deconvoluted XPS spectra of C 1s (b), N 1s (c), O 1s (d), Si 2p (e), S 2s (f) and S $2 p$ (g). 

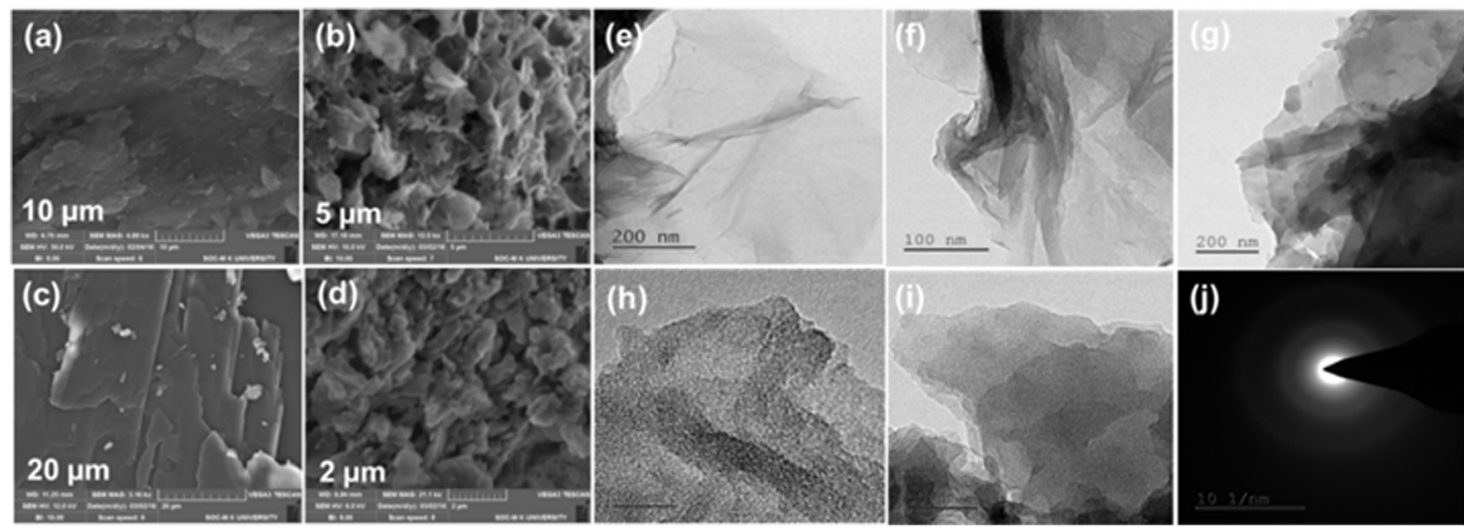

Fig. 3 SEM images of GO (a), GOP (b), GOPN (c), GOPND (d), HR-TEM images GO (e), GOP (f), GOPN (g), GOPND (h and i) and SAED pattern of GOPND (j).

influential analytes. The presence of the bis(pyridyl) motif in GOPND is expected to be an analyte binding site, with the luminescent dansyl motif as a signalling unit, and both are synchronized together and anticipated to give a good PL response through the energy transfer pathway. The as-prepared GOPND has good dispersibility in water, which gives an excellent UV-vis spectrum with three absorption bands at 214, 268, and $369 \mathrm{~nm}$ (Fig. 4). Its respective precursor GOP showed absorption at 265 and $287 \mathrm{~nm}$, and successive silylated GOPN gave peaks at 229 and $258 \mathrm{~nm}$. When compared to electronic spectra (Fig. S5, ESI $\dagger$ ) of all precursors and free organic motifs bis(pyridyl) and dansyl chloride, after functionalization, the three peaks of GOPND were red-shifted to longer wavelength owing to the energy transfer capability of GO; this is further evidence of covalent functionalization. In the solubility studies of GOPND with different solvents, a more stable dispersion was observed in water than in other organic solvents; hence all absorption and emission studies were carried out in an aqueous phosphatebuffered saline (PBS) buffer medium to maintain the $\mathrm{pH}$ at 7 .

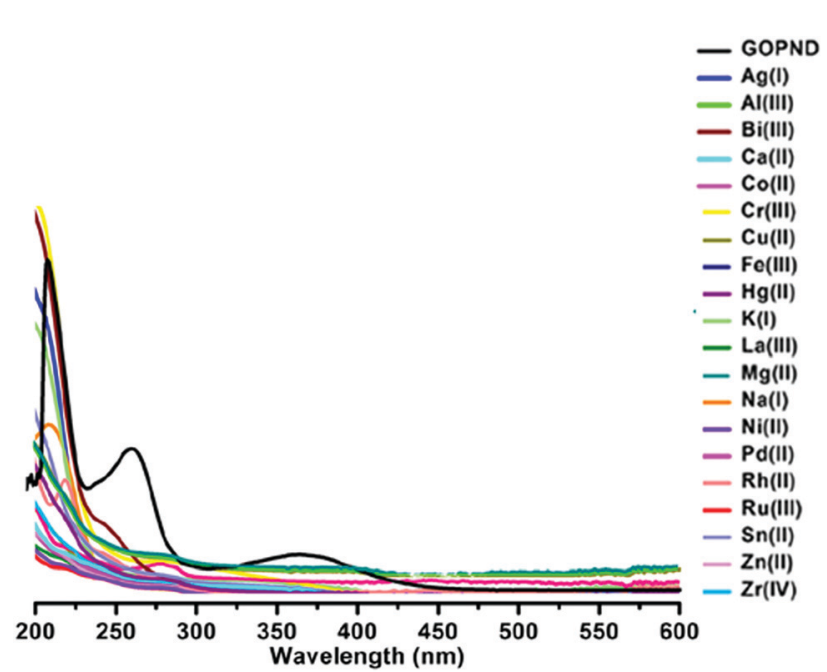

Fig. 4 UV-vis spectra of GOPND (10 ppm) with various metal cations (Ag(I), Al(III), Bi(III), Ca(II), Co(II), Cr(II), Cu(II), Fe(III), Hg(II), K(I), La(III), Mg(II), Na(I),

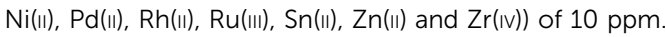

In general, dansyl chloride is insoluble in water; however, the covalently functionalized GOPND offers a better dispersibility and also enhances the solubility of dansyl, further resulting in an excellent green, fluorescence when exited at $365 \mathrm{~nm}$. Fig. S6 reveals the emission spectra of PAE, GOP, GOPN, DCL and GOPND at $\lambda_{\mathrm{ex}}=370 \mathrm{~nm}$. Further to understand the absorption response of GOPND, it was studied with a series of metal ions, including alkali, alkaline earth, p-block, transition, and rare earth metals with different oxidation states, such as $\mathrm{Ag}(\mathrm{I})$, $\mathrm{Al}(\mathrm{III}), \mathrm{Bi}$ (III), $\mathrm{Ca}$ (II), $\mathrm{Co}$ (II), $\mathrm{Cr}$ (III), $\mathrm{Cu}$ (II), $\mathrm{Fe}$ (III), $\mathrm{Hg}$ (III), K(I), La(III), $\mathrm{Mg}(\mathrm{II}), \mathrm{Na}$ (I), Ni(II), Pd(II), Rh(II), Ru(III), Sn(II), Zn(II) and Zr(Iv). But none of the metal ions showed any distinct response to the GOPND probe, and there was no change in the absorption intensity; however, a blue-shift was noted.

Further, to explore the interaction of metal ions with the probe, an absorption titration was carried out using GOPND $(10 \mathrm{ppm})$ with a random concentrations of metal ions $(10,100$, and $200 \mathrm{ppm}$ ) in which, except for Ru(III), no metal ion showed any response (Fig. S7, ESI $\dagger$ ). Whereas after the addition of $100 \mathrm{ppm}$ of $\mathrm{Ru}(\mathrm{III})$, a visual enhancement was found with a blueshifted absorption band at $281 \mathrm{~nm}$, and it continued with $150 \mathrm{ppm}$. With these encouraging results, a complete titration using 10-200 ppm of $\mathrm{Ru}(\mathrm{III})$ was carried out (Fig. 5), and an apparent enhancement of the blue-shifted bands at $281 \mathrm{~nm}$ was observed, and, further, it gets shifted to $276 \mathrm{~nm}$. The change in the absorption is rationalized by the coordination of $\mathrm{Ru}(\mathrm{III})$ with bis(pyridyl), which arrests the energy transfer to the dansyl motif in the ground state, which is also revealed by the absence of a visual colour change. The preliminary absorption studies showed a notable change while interacting with $\mathrm{Ru}(\mathrm{III})$. However, it does not provide any informative results regarding the sensing of $\mathrm{Ru}(\mathrm{III})$ ions. To develop a reliable sensing methodology further, we moved on to fluorescence studies. The PL studies of GOPND were carried out in an aqueous PBS buffer. Here it is essential to remember that GO and its derived materials do not obey Kasha's rule ${ }^{78}$ in polar solvents; they exhibit a giant red-edge shift. To find the best excitation wavelength for GOPND in the polar PBS medium, the screened wavelengths were 360,370 , and $380 \mathrm{~nm}(10 \mathrm{ppm})$, and that at 


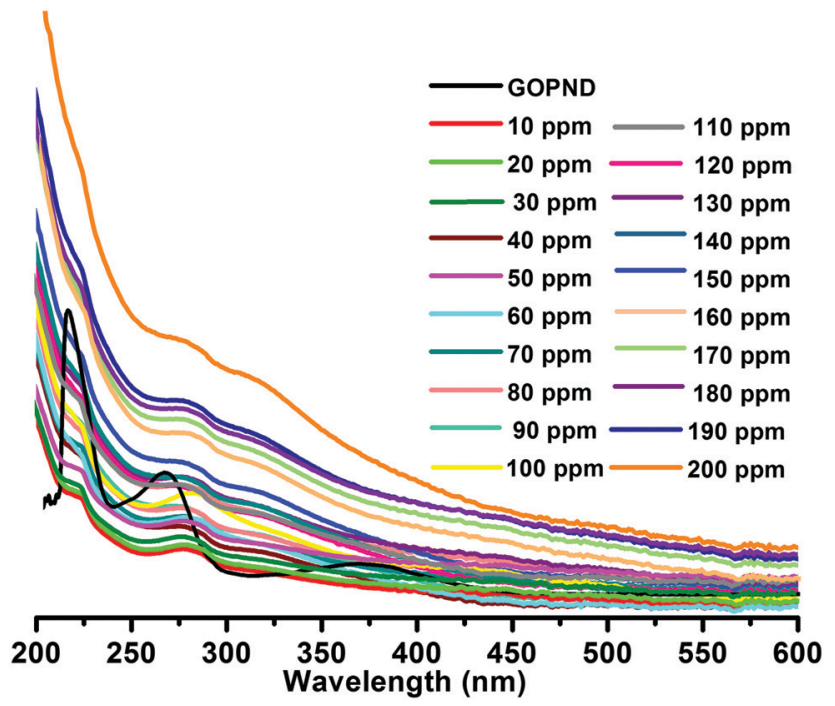

Fig. 5 UV-vis spectral titration of GOPND (10 ppm) with Ru(III) (10-200 ppm) at $\mathrm{pH} 7$.

$370 \mathrm{~nm}$ showed an excellent PL response in $519 \mathrm{~nm}$ with a maximum intensity in minimum concentration (Fig. S8, ESI $\dagger$ ).

Under a similar excitation wavelength except for dansyl chloride, other precursors PAE, GOP, and GOPN are nonemissive (Fig. S6, ESI $\dagger$ ). The PL intensity of GOPND was slightly decreased compared to the free dansyl chloride under identical conditions owing to the quenching efficiency of GO.

With the above-screened excitation wavelength, all the metal ions tested in absorption studies, such as $\mathrm{Ag}$ (I), $\mathrm{Al}(\mathrm{III}), \mathrm{Bi}(\mathrm{III})$, $\mathrm{Ca}$ (II), $\mathrm{Co}$ (II), $\mathrm{Cr}$ (III), $\mathrm{Cu}$ (II), $\mathrm{Fe}$ (III), $\mathrm{Hg}$ (II), $\mathrm{K}$ (I), $\mathrm{La}$ (III), $\mathrm{Mg}$ (II), $\mathrm{Na(I)}$, $\mathrm{Ni}(\mathrm{II}), \mathrm{Pd}(\mathrm{II}), \mathrm{Rh}$ (II), Ru(III), Sn(II), Zn(II) and $\mathrm{Zr}$ (IV), were excited with GOPND. Unlike the absorption results, the PL studies showed a distinct response, where all the tested metal ions were highly inert without any influence on the PL spectra of GOPND (Fig. S9, ESI $\dagger$ ) except Ru(III).

Treating 10 ppm of Ru(III) with 10 ppm of GOPND showed an immediate fluorescence quenching when excited at $370 \mathrm{~nm}$. Even with higher concentrations (10 ppm, 100 ppm and 200 ppm) (Fig. 6) none of the other metals quenches the fluorescence enhancement of GOPND (Fig. S9, ESI $\dagger$ ).

This reveals that no metal ion other than $\mathrm{Ru}(\mathrm{III})$ has any remarkable response towards GOPND. The images of GOPND under a UV lamp in the presence of different metal cations are a visual proof of selective fluorescence quenching for $\mathrm{Ru}(\mathrm{III})$ (Fig. 7). This reveals the response of $\mathrm{Ru}(\mathrm{III})$ cations towards GOPND. None of the other cations shows any quenching; rather they slightly enhance the fluorescence over $\mathrm{Ru}(\mathrm{III})$. Ru(III) selectively quenches the PL intensity. Even the increased concentration of metal ions does not vary the intensity as like $\mathrm{Ru}(\mathrm{III})$ and seldom shows a response, whereas the decreasing and increasing concentration of $\mathrm{Ru}(\mathrm{III})$ gives respective PL spectra as well as in naked eye detection studies under UV light (Fig. 7). Further, to explore the sensing behaviours of GOPND, a titration was carried out. During the sequential addition of Ru(III) with an increasing 10 ppm scale, complete quenching was noted after the addition of

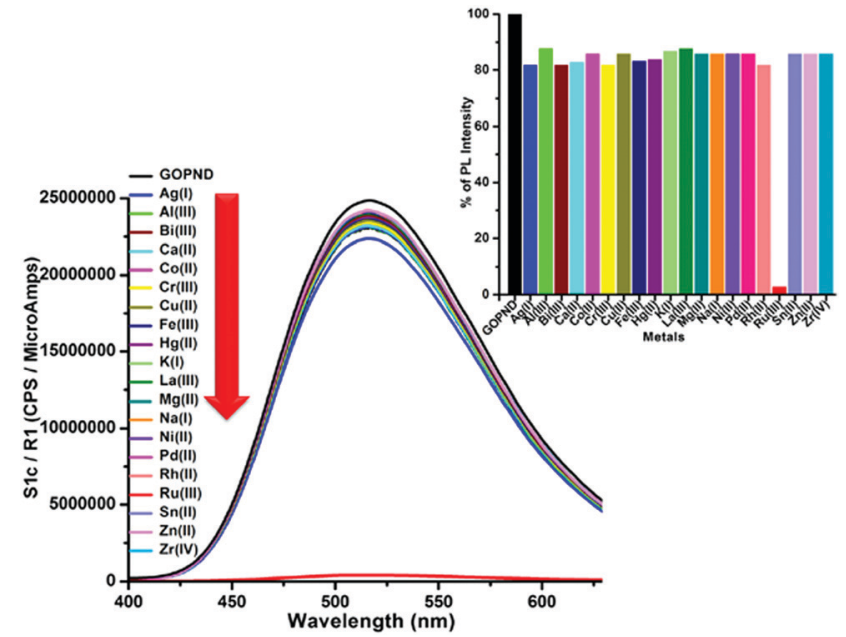

Fig. $6 \mathrm{PL}$ spectral changes of GOPND ( $c=10 \mathrm{ppm}$ ) in the presence of

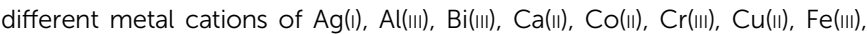

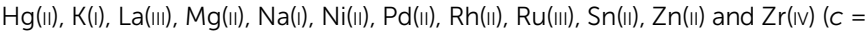
$200 \mathrm{ppm}$ ) at $\lambda_{\mathrm{ex}}=370 \mathrm{~nm}$. (Inset: histogram representing the PL emission intensity of GOPND with different analytes while exciting to $370 \mathrm{~nm}$ )

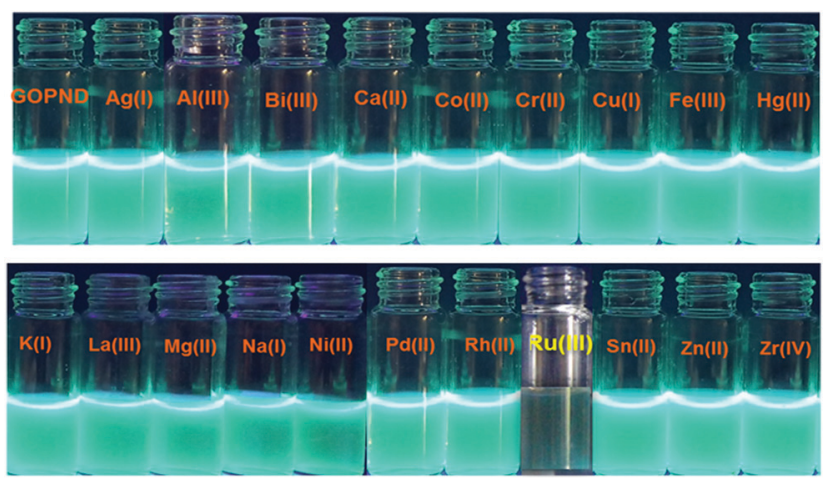

Fig. 7 Images of GOPND with different metal cations from $\mathrm{Ag}(\mathrm{I}), \mathrm{Al}(\mathrm{III})$,

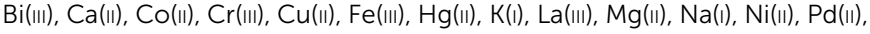
$\mathrm{Rh}(\mathrm{II}), \mathrm{Ru}(\mathrm{III}), \mathrm{Sn}(\mathrm{II}), \mathrm{Zn}(\mathrm{II})$ and $\mathrm{Zr}(\mathrm{IV})$ in $\mathrm{pH}-7$ under a UV-lamp (365 nm).

200 ppm. As observed in Fig. 8, an immediate quenching in the PL response of the GOPND in the presence of $\mathrm{Ru}(\mathrm{III})$ at $\lambda_{\mathrm{ex}}=370 \mathrm{~nm}$ strongly represents the high selectivity of GOPND towards the detection of $\mathrm{Ru}(\mathrm{III})$ in the aqueous medium.

Though the GOPND showed excellent selectivity by decreasing its PL intensity with $\mathrm{Ru}(\mathrm{III})$, the selectivity, sensitivity, and the competitive sensing potential of the probe in the presence of other common metal ions were also investigated, and the results are presented in Fig. 9.

Among all the screened metal ions, the quenching response of $\mathrm{Ru}(\mathrm{III})$ ions with GOPND was unique and incomparably higher. During the competitive studies between $\mathrm{Ru}(\mathrm{III})$ with other metal ions, on the addition of different metal ions, even with a higher concentration of $50 \mathrm{ppm}$ together with $50 \mathrm{ppm}$ of $\mathrm{Ru}(\mathrm{III})$, no notable change in the quenching behavior was shown; indeed, the actual PL quenching nature of $\mathrm{Ru}(\mathrm{III})$ with GOPND was retained up to $2 \%$. 


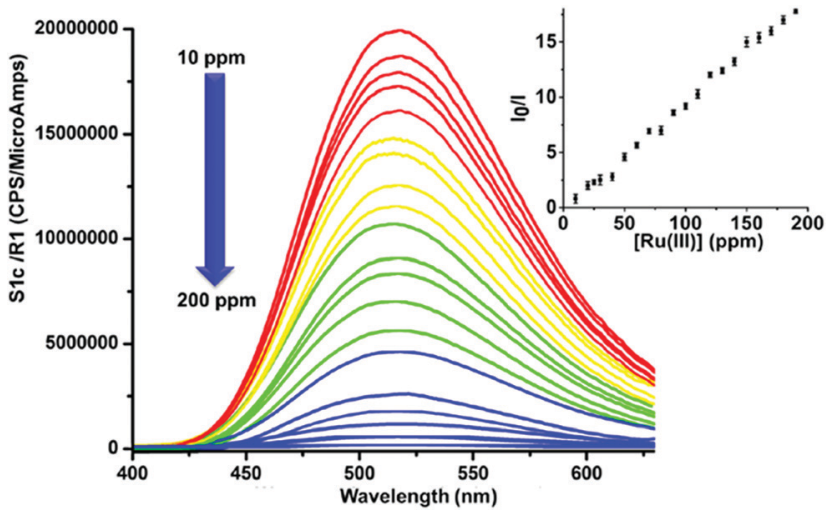

Fig. 8 PL spectral changes of GOPND (10 ppm) titrated against Ru(III) (10$200 \mathrm{ppm}$ ) with $10 \mathrm{ppm}$ increment at $\mathrm{pH} 7, \lambda_{\mathrm{ex}}=370 \mathrm{~nm}$ (inset: calibration curve, where $I_{0}$ - initial intensity, $I$ - intensity after the addition of Ru(III)).

The same response was retained even after the concentration of the other metal ion was raised to $110 \mathrm{ppm}$. Meanwhile, some of the other metal ions were only slightly influenced by the quenching efficiency of $\mathrm{Ru}(\mathrm{III})$. Metal ions such as $\mathrm{Cu}(\mathrm{II})$ and $\mathrm{Rh}$ (II) ions quenched the PL intensity around $19-20 \%$ along with $\mathrm{Ru}(\mathrm{III})$ ions; however, this behaviour was not shown when those ions were individually titrated. Nevertheless, the competitive quenching behaviour of other metal ions does not influence the selectivity of $\mathrm{Ru}(\mathrm{III})$ with GOPND. The competitive studies unambiguously demonstrate that GOPND behaves as an efficient and specific sensing probe for $\mathrm{Ru}(\mathrm{III})$ ions, even in the presence of other metal ions with excess concentration.

In the development of the materials as sensing probes, a study of the $\mathrm{pH}$ of the medium is inevitable. It is one of the decisive parameters to fine-tune the sensing potential of the developed sensor probe. The as-prepared GOPND has several nitrogen centers in the bis(pyridyl) and dansyl motifs; thus, understanding the role of $\mathrm{pH}$ in the sensing medium is

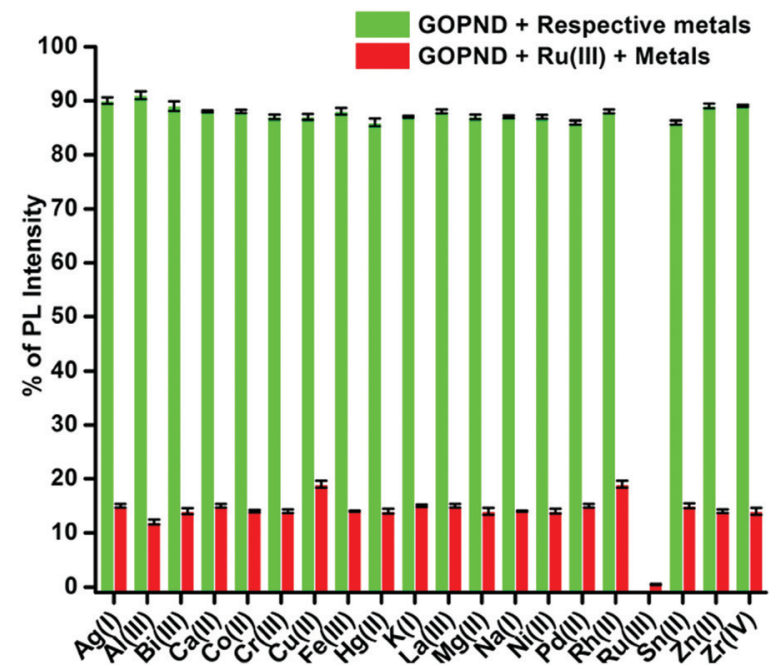

Fig. 9 Competitive studies: fluorescence response of GOPND ( $c=10$ ppm) Green bars: 50 ppm addition of other metal ions; red bars: 50 ppm addition of other metal ions along with 50 ppm addition of Ru(II) in PBS buffer at $\mathrm{pH} 7$.

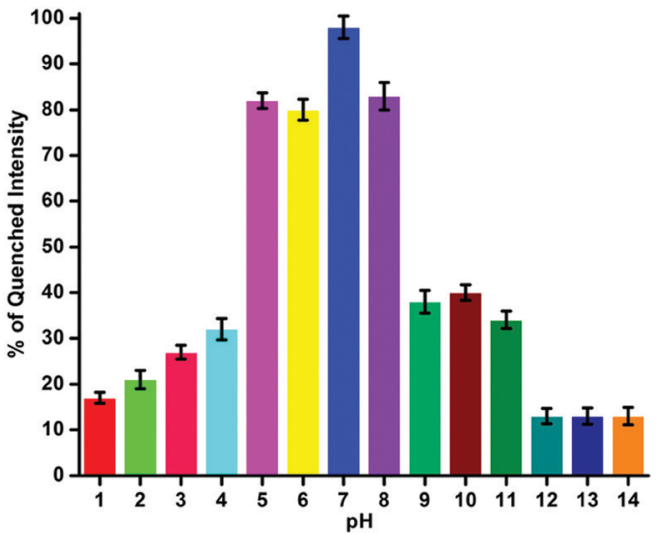

Fig. 10 Fluorescence response of the GOPND (10 ppm) toward Ru(III) (200 ppm) under different $\mathrm{pH}$ conditions.

essential. The sensing behaviours of the GOPND probe with $\mathrm{Ru}(\mathrm{III})$ in different $\mathrm{pH}$ ranges from 1 to 14 were studied by tuning the buffer in an aqueous medium. The observed PL quenching response of GOPND in the presence of $\mathrm{Ru}(\mathrm{III})$ under different $\mathrm{pH}$ ranges have been presented in Fig. 10. The analysis of the $\mathrm{pH}$ response plot (Fig. 10) disclosed that the change in the $\mathrm{pH}$ directly influences the probe's sensing response. When the $\mathrm{pH}$ is decreased towards the acidic, the quenching process starts falling. At pH 5, 6 and 7, the probe is not much influenced, and only an 8 to $18 \%$ decrease in the quenching efficiency was noted. However, after $\mathrm{pH} 4$, a sharp reduction of around 68 to $83 \%$ in the probe's quenching efficiency was observed. This behavior is rationalized through the protonation of the nitrogens present on GOPND, blocking the binding sites of $\mathrm{Ru}(\mathrm{III})$. On the other hand, a similar trend was repeated when the $\mathrm{pH}$ increased to 8 , and a similar decrease in the quenching efficiency was observed up to $\mathrm{pH}$ 11. After $\mathrm{pH} \mathrm{12}$, a sudden drop in the quenching behavior of GOPND was observed and only $13 \%$ quenching was noted up to the higher $\mathrm{pH}$ 14. These quenching studies of the probe under different $\mathrm{pH}$ conditions and the changes in its efficiency strongly endorse that the prepared GOPND probe's sensing behaviour has been influenced by $\mathrm{pH}$ and only under physiologically neutral $\mathrm{pH}$ is more responsive.

Unlike homogeneous chemosensors, probes derived from carbon-based materials are highly stable and retain their PL capability for a long time without suffering any chemical or physical changes. The present GOPND probe is a graphenederived material and heterogeneous in nature. Hence to understand the stability and PL response of the prepared GOPND probe in PBS buffer media, fluorescence emission intensity and peak shapes were monitored with respect to time. With the probe alone fluorescence intensity was periodically checked in $12 \mathrm{~h}$ intervals, revealing that the GOPND is stable in PBS buffer even after $48 \mathrm{~h}$ without any remarkable change in peak shape or emission intensity (Fig. 11).

In addition, the detection ability of the GOPND probe was also monitored using the same stock solution prepared $48 \mathrm{~h}$ before, and a negligible change in the sensing behavior was 


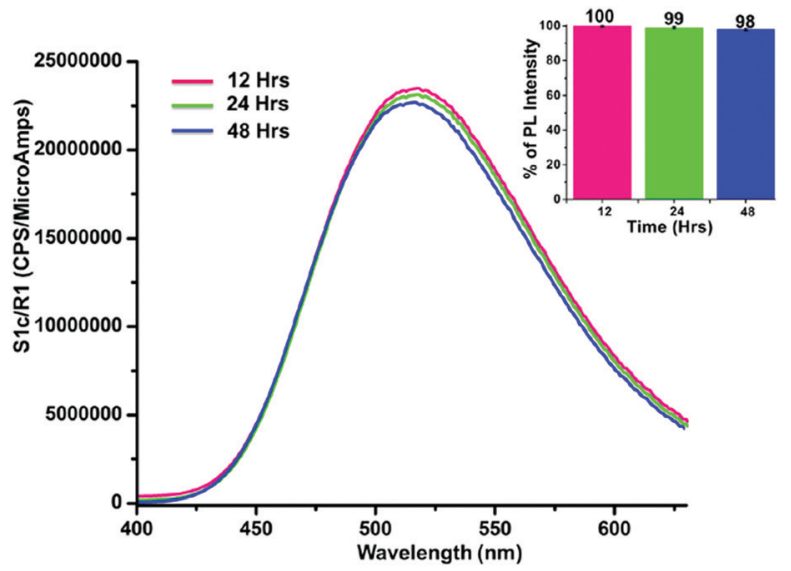

Fig. 11 Fluorescence response stability study of the GOPND probe at different time intervals $(12,24$, and $48 \mathrm{~h}), \lambda_{\mathrm{ex}}=370 \mathrm{~nm}$. Inset: Bar-diagram of fluorescence response with respect to time.

observed (Fig. 12). Both the studies strongly endorse the stability of the GOPND probe in the aqueous medium in $\mathrm{Ru}(\mathrm{III})$ detection, which is extremely helpful in using this sensor probe for periodical applications.

Next, to understand the necessity and significance of the covalent modifications on the GO basal plane in the present $\mathrm{Ru}(\mathrm{III})$ sensor study, control experiments were pursued using the corresponding precursors used to prepare GOPND. A separate PL study of GO, PAE, and dansyl chloride showed that none of them interact with $\mathrm{Ru}(\mathrm{III})$ even at high concentration (200 ppm) and showed no response except for GOPND and Ru(III) (10 ppm) (Fig. S10, ESI $\dagger$ ). Further, another successive precursor, GOP, in which the bis(pyridyl) is attached to GO through an ester bond, is highly inert towards $\mathrm{Ru}(\mathrm{III})$ without emitting any PL response when excited at $370 \mathrm{~nm}$. Meantime, the same GOP probe is highly responsive towards $\mathrm{Zr}(\mathrm{Iv})$ and explored as a turn-on fluorescence sensor. ${ }^{79}$ A similar inert response was also observed with GOPN (Fig. S10, ESI $\dagger$ ).

All the above control studies clearly confirmed that complete functionalization of GO with bis(pyridyl) and dansyl motifs and

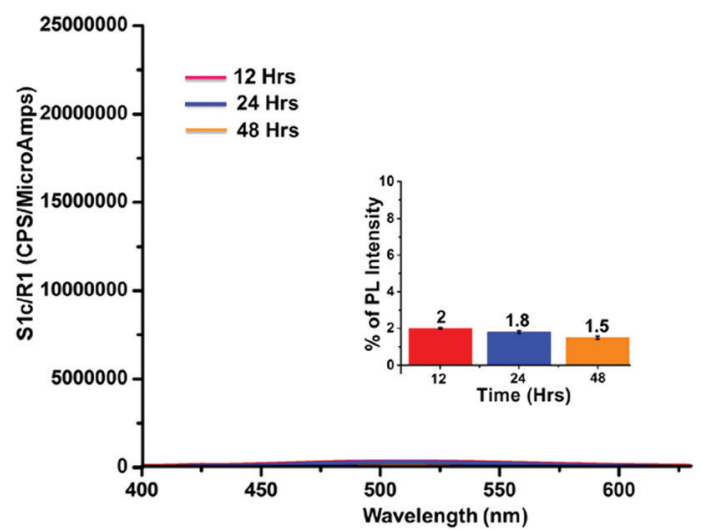

Fig. 12 Fluorescence response stability study of the GOPND probe with $\mathrm{Ru}(\mathrm{III})$ at different time intervals $(12,24$, and $48 \mathrm{~h}), \lambda_{\mathrm{ex}}=370 \mathrm{~nm}$. Inset: Bardiagram of fluorescence response with respect to time. the resulting covalently modified GOPND could act as a responsive turn-off sensor only for $\mathrm{Ru}(\mathrm{III})$. In another study, a physical mixture of GO (2.5 ppm), PAE (2.5 ppm), APTES (2.5 ppm), and dansyl chloride (2.5 ppm) along with $\mathrm{Ru(III)}$ (100 ppm) was tested and the physical mixture did not show any fluorescence quenching.

This reveals that the physical mixture does not exhibit the fluorescence of GOPND and furthermore the quenching behavior does not originate from the non-covalent binding or physisorption (Fig. S11, ESI $\dagger$ ). In another study, to understand the fate of GOPND after Ru(III) sensing, the used probe was collected and recovered by centrifugation. The recovered probe was repeatedly washed with water and dialyzed three times in DD to remove the $\mathrm{Ru}(\mathrm{III})$ ions and finally washed with dry acetone. The complete removal of ruthenium ions and the absence of other species in the recovered GOPND was confirmed by the absorption and emission analysis of the filtrate. Additionally, it was dried under vacuum for $24 \mathrm{~h}$ and subjected to FT-IR analysis. The FT-IR study demonstrates that the GOPND probe is disturbed after the sensing process and the dansyl motif is cleaved from GOPND. The absence of the peaks at 785, 1210, 1473, 1390, and $2491 \mathrm{~cm}^{-1}$ corresponding to the dansyl motif revealed the probe's cleavage, and the FT-IR of the recovered samples agreed with that of GOPN. So, after the sensing process was complete, the recovery process may disrupt the sulfonyl amide bonding of GOPND, albeit the rest of the functionalization (GOPN) is not affected (Fig. S12, ESI $\dagger$ ). To examine the application of the developed GOPND probe in real-time applications, further studies were carried with the samples collected from natural sources, an aqueous workup of ruthenium-catalyzed reaction, and laboratory effluent. In synthetic organic chemistry and catalysis research, rutheniumbased catalysts have been widely consumed. Notably, rutheniumderived homogeneous catalysts ${ }^{80}$ have gained wide application, and after reaction completion, they are discarded as waste. To detect ruthenium from such effluent, we chose three samples to examine the sensing potential of GOPND: the aqueous workup from an $\mathrm{RuCl}_{3}$ derived asymmetric catalysis reaction, and another sample collected from an academic research laboratory drain. One more water sample was collected from the Vaigai riverbed of India. The collected samples were filtered through a $2 \mu$ filter, and only the resulting clear aqueous solution was taken for analysis. For the real sample analysis, a 1:1 ratio of GOPND and samples was taken under the optimized identical conditions and excited at $\lambda_{\text {ex }}=370 \mathrm{~nm}$. Both the samples collected from laboratories showed an impressive response and a significant quenching in the PL intensity of GOPND (Fig. 13). Meantime a weak response was found from the river water samples. A comparative study of the real-time samples with standard titration measurement shows the presence of 110,100 , and $20 \mathrm{ppm}$ of $\mathrm{Ru}(\mathrm{III})$ from aqueous workup, laboratory drain, and river water samples, respectively.

The observed results from the real-time samples were also cross-verified with the ICP-OES analysis, and the results agreed with the GOPND probe result within $\pm 2 \%$ error limit. The response and results from the real-time samples strongly ascertain the GOPND probe's efficiency in the detection of $\mathrm{Ru}(\mathrm{III})$. The Stern-Volmer plot explains the quenching constant: 


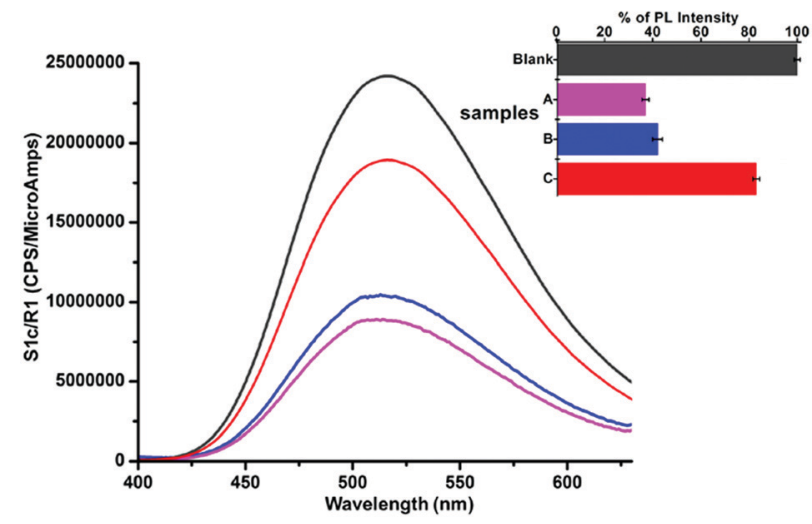

Fig. 13 Fluorescence spectra of GOPND with different real samples in the presence and absence of $\mathrm{Ru}\left(\mathrm{III}\right.$ ) (inset: the corresponding histogram), $\lambda_{\mathrm{ex}}=$ $370 \mathrm{~nm}$ (blank, GOPND; A - aqueous waste from asymmetric catalysis work-up; B - research laboratory drain-out; C - water sample from Vaigai river).

it is more significant for $\mathrm{Ru}(\mathrm{III})$ since $K_{\mathrm{sv}}=9.9 \times 10^{3} \mathrm{M}^{-1}$ (Fig. S13, ESI $\dagger$ ). The LOD value was calculated from the SternVolmer plot using the standard formula $(3 \sigma / k)$, where ' $\sigma$ ' is the concentration of $\mathrm{Ru}(\mathrm{III})$ and ' $k$ ' is the slope obtained from the Stern-Volmer plot. ${ }^{81}$ Using the above equation, the LOD of the GOPND probe was calculated as $15 \mathrm{nM}$. The sensing ability of the GOPND towards $\mathrm{Ru}(\mathrm{III})$ through fluorescence quenching is rationalized by proposing a mechanism based on literature reports. ${ }^{19,82,83}$ In general, GO exhibits excellent emission properties owing to the presence of an extended conjugation system and diverse oxygen functionalities; however, in most of the PL studies, due to the charge transfer process, the fluorescence response was quenched by various mechanistic pathways and positioned as a turn-off sensor. With a similar strategy, the present highly fluorescent GOPND covalently functionalized with bis(pyridine) and dansyl motifs acts as a turn-off sensor when it selectively binds with $\mathrm{Ru}(\mathrm{III})$. Here, the GO basal plane provides a better platform for electron-hole recombination, which is induced by the electronic transitions between the non-oxidized carbon atom regions and oxidized carbon atom regions. The prepared GOPND probe was designed based on the energy transfer phenomenon, where the energy acceptor and donor are separated by the Förster energy distance. When the silyloxy propyl unit acts as a spacer between bis(pyridine) functionalized GO and the dansyl motif, it all comes into close proximity. From the literature, ${ }^{84}$ it is understood that the dansyl motif acts as an energy donor, and the bis(pyridine) unit is positioned as an acceptor in GOPND. GOPND itself emits green fluorescence at $365 \mathrm{~nm}$ in an aqueous medium owing to the presence of the dansyl motif.

When excited at $370 \mathrm{~nm}$, the excitation wavelength of dansyl chloride, it gives maximum fluorescence enhancement in an aqueous medium which is due to the giant red edge effect of GO. (It is possible only due to the functionalization of dansyl with GO; hence the dansyl motif alone has low or no fluorescence under an aqueous medium.) Meanwhile, the addition of the $\mathrm{Ru}(\mathrm{III})$ readily and selectively binds with the bis(pyridine) owing to the existence of a pseudo-cavity caused by two pyridinic nitrogens and one aliphatic tertiary nitrogen (hard base). When GOPND is excited, after the addition of $\mathrm{Ru}(\mathrm{III})$, a synchronized energy transfer from the dansyl motifs to the $\mathrm{Ru}(\mathrm{III})$ bound bis(pyridine) would take place through Förster resonance energy transfer. After the energy transfer from the dansyl unit to the $\mathrm{Ru}(\mathrm{III})$ bound bis(pyridine), there is a lack of sufficient energy required for the free rotation of the $N, N$-dimethyl group of the dansyl motif and the restricted conjugation of the lone pair of electrons decreases the fluorescence intensity and results in quenching. The same quenching behavior could also be possible by other quenching mechanistic pathways like ICT and PET; however, since the energy donor dansyl and acceptor bis(pyridyl) are present in the same GO domain as a single molecule, owing to the lack of extended conjugation, other energy transfer mechanisms are not possible. The same behavior continued when the concentration of the Ru(III) was increased and the PL of GOPND was completely quenched after the addition of $200 \mathrm{ppm}$ of $\mathrm{Ru}(\mathrm{III})$. On the other hand, in order to understand the role of the covalently functionalized GO in the present Ru(III) detection process, a controlled study was carried with a $1: 1: 1$ physical mixture of the GO, 2-bis(pyridine-2-ylmethyl) amino)ethan-1-ol and dansyl chloride, which did not show any fluorescence quenching response under identical study conditions (Fig. S11, ESI $\dagger$ ). This control study strongly ascertained the importance of the covalently functionalized GOPND, where the bis(pyridine) and dansyl motif are brought into proximity on the graphitic domain and facilitate the FRET-based energy transfer to detect $\mathrm{Ru}(\mathrm{III})$ in the aqueous medium. Further the efficiency and sensitivity of the present GOPND probe is compared with previously reported $\mathrm{Ru}(\mathrm{III})$ detection methodologies (Table S1, ESI $\dagger$ ). According to Table S1 (ESI $\dagger$ ), so far reported probes show LOD in the region of micro-molar concentrations and very few reports show the nanomolar region; also the sustainability is still questionable and there was a failure to explain the sensing mechanism. Here GOPND has shown reliable results with good sensitivity in a nanomolar detection range and has more advantages than other probes. The present carbonaceous sensor material has an LOD of $15 \mathrm{nM}$ in a complete aqueous medium.

\section{Conclusion}

A novel and carbonaceous sensor material GOPND was synthesized through covalently functionalizing pristine graphene oxide with PAE through Steglich esterification on the edges and dansyl chloride on the basal plane. Three-step functionalization promisingly provides a better PL response with $\mathrm{Ru}(\mathrm{III})$. The synthesized GOPND was thoroughly characterized using various possible and feasible analytical techniques, which confirmed the covalent functionalization. The enhanced PL properties of the synthesized GOPND material in the aqueous medium have been utilized as a turn-off chemosensor for the detection of industrially and academically essential ruthenium(III) ions at physiological $\mathrm{pH}$ with the lowest detection limit of $15 \mathrm{nM}$ (31 ng $\mathrm{mL}^{-1}$ or $\left.31 \mathrm{ppb}\right)$. The efficient 
fluorescence quenching response of GOPND unveils high selectivity towards $\mathrm{Ru}(\mathrm{III})$ even in the presence of other common metal ions. The selective sensing behavior of GOPND towards $\mathrm{Ru}(\mathrm{III})$ is explained through the FRET mechanism owing to the presence of both bis(pyridyl) and dansyl motifs in proximity through the covalent functionalization of GO. The real-time application of GOPND was proved by detecting $\mathrm{Ru}(\mathrm{III})$ from laboratory effluent and river water. The development of such carbon material-based sensing methodology and selective detection ability from covalently functionalized graphene materials finds wide applications in developing a carbonaceous and straightforward material-based sensor system for electronics and chemical applications.

\section{Author contributions}

Preparations of materials, material characterization, manuscript preparation and analysis of results, J. B. Asha; formal analysis, assisting for the preparation of materials, M. Karthik; conceptualization, investigation, manuscript preparation and supervision, Dr P. Suresh.

\section{Conflicts of interest}

There are no conflicts to declare.

\section{Acknowledgements}

We gratefully acknowledge the financial support from the Science and Engineering Research Board (SERB), New Delhi, India, (Grant No. SR/FT/CS-53/2011), University Grants Commission, New Delhi, India, (UGC grant No.: 42-291/ 2013(SR)), India and Council for Scientific and Industrial Research, New Delhi, India (CSIR grant No.: 02(0191)14/EMR-II). We thank University Grants Commission under University with Potential for Excellence program (UGC-UPE) and HR-TEM, DST-FIST (for LC-MS) and DST-PURSE (for FT-IR, SEM, and EDX) for the instrumental facility.

\section{Notes and references}

1 B. Majumdar, D. Sarma, T. Bhattacharya and T. K. Sarma, ACS Sustainable Chem. Eng., 2017, 5, 9286.

2 A. T. Smith, A. M. LaChance, S. Zeng, B. Liu and L. Sun, Nano Mater. Sci., 2019, 1, 31.

3 Y. Sheng, X. Tang, E. Peng and J. Xue, J. Mater. Chem. B, 2013, 1, 512.

4 Y. Chen, Y. Yang, Y. Xian, P. Singh, J. Feng, S. Cui, A. Carrier, K. Oakes, T. Luan and X. Zhang, ACS Appl. Mater. Interfaces, 2020, 12, 352.

5 J. G. Radich and P. V. Kamat, ACS Catal., 2012, 2, 807.

6 Y. V. Stebunov, O. A. Aftenieva, A. V. Arsenin and V. S. Volkov, ACS Appl. Mater. Interfaces, 2015, 7, 21727.

7 X.-M. Huang, L.-Z. Liu, S. Zhou and J.-J. Zhao, Front. Phys., 2020, 15, 33301.
8 D. R. Dreyer, S. Park, C. W. Bielawski and R. S. Ruoff, Chem. Soc. Rev., 2010, 39, 228.

9 E. Jaafar, M. Kashif, S. K. Sahari and Z. Ngaini, Mater. Sci. Forum, 2018, 917, 112.

10 T.-F. Yeh, C.-Y. Teng, L.-C. Chen, S.-J. Chen and H. Teng, J. Mater. Chem. A, 2016, 4, 2014.

11 M. T. Hasan, B. J. Senger, C. Ryan, M. Culp, R. G. Rodriguez, J. L. Cofer and A. V. Naumov, Sci. Rep., 2017, 7, 6411.

12 Z. Li, C. He, Z. Wang, Y. Gao, Y. Dong, C. Zhao, Z. Chen, Y. Wu and W. Song, Photochem. Photobiol. Sci., 2016, 15, 910.

13 A. Pandiyarajan, D. J. Dmonte, N. Bhuvanesh, S. Suresh, B. Vidhya and R. Nandhakumar, J. Nanosci. Nanotechnol., 2019, 19, 5189.

14 P.-J. J. Huang and J. Liu, Anal. Chem., 2012, 84, 4192.

15 V. Georgakilas, J. N. Tiwari, K. C. Kemp, J. A. Perman, A. B. Bourlinos, K. S. Kim and R. Zboril, Chem. Rev., 2016, 116, 5464.

16 R. Liu, J. Hu, S. Zhu, J. Lu and H. Zhu, ACS Appl. Mater. Interfaces, 2017, 9, 33029.

17 H.-J. Kim, J. Sung, H. Chung, Y. J. Choi, D. Y. Kim and D. Kim, J. Phys. Chem. C, 2015, 119, 11327.

18 S. Ge, F. Lan, F. Yu and J. Yu, New J. Chem., 2015, 39, 2380. 19 X. Wu, Y. Xing, K. Zeng, K. Huber and J. X. Zhao, Langmuir, 2018, 34, 603.

20 J. Kim, L. J. Cote, F. Kim and J. Huang, J. Am. Chem. Soc., 2010, 132, 260.

21 Z. Liu, Q. Liu, Y. Huang, Y. Ma, S. Yin, X. Zhang, W. Sun and Y. Chen, Adv. Mater., 2008, 20, 3924.

22 H. Dong, W. Gao, F. Yan, H. Ji and H. Ju, Anal. Chem., 2010, 82, 5511.

23 P. Zheng and N. Wu, Chem. - Asian J., 2017, 12, 2343.

24 L. Zhang, D. Peng, R.-P. Liang and J.-D. Qiu, Trends Anal. Chem., 2018, 102, 280-289.

25 D. J. Dmonte, A. Pandiyarajan, N. Bhuvanesh, S. Suresh and R. Nandhakumar, Mater. Lett., 2018, 227, 154-157.

26 Y. Yaoa, G. Wanga, G. Chu, X. Ana, Y. Guo and X. Sun, New J. Chem., 2019, 43, 13816-13826.

27 Y. Wang, L. Tang, Z. Li, Y. Lin and J. Li, Nat. Protoc., 2014, 9, 1944-1955.

28 J. Liu, G. Liu, W. Liu and Y. Wang, Biosens. Bioelectron., 2015, 64, 300-305.

29 H. Zhang, L. Feng, B. Liu, C. Tong and C. Lü, Dyes Pigm., 2014, 101, 122-129.

30 S. Ebrahim, A. Shokry, M. M. A. Khalil, H. Ibrahim and M. Soliman, Sci. Rep., 2020, 10, 13617-13627.

31 A. Shokry, M. M. A. Khalil, H. Ibrahim, M. Soliman and S. Ebrahim, Sci. Rep., 2019, 9, 16984-16995.

32 A. Shokry, A. E. Tahan, H. Ibrahim, M. Soliman and S. Ebrahim, RSC Adv., 2019, 9, 39187-39200.

33 B. Neises and W. Steglich, Angew. Chem., Int. Ed. Engl., 1978, 17, 522 .

34 C. S. Allardyce and P. J. Dyson, Platinum Met. Rev., 2001, 45, 62. 35 S. Megremis and C. M. Carey, Corrosion and tarnish of dental alloys, American Society of Metals (ASM), 2017 Handbook, $13 \mathrm{~B}$. 
36 L. Messori, M. Camarri, T. Ferraro, C. Gabbiani and D. Franceschini, ACS Med. Chem. Lett., 2013, 4, 329.

37 A. B. Filonov, D. B. Migas, V. L. Shaposhnikov, N. N. Dorozhkin, V. E. Borisenko, A. Heinrich and H. Lange, Phys. Rev. B: Condens. Matter Mater. Phys., 1999, 60, 16494.

38 A. Islam, S. P. Singh and L. Han, Int. J. Photoenergy, 2011, 204639.

39 C. Creutz and N. Sutin, Proc. Natl. Acad. Sci. U. S. A., 1975, 72, 2858.

40 R. H. Grubbs, Angew. Chem., Int. Ed., 2006, 45, 3760.

41 R. R. Schrock, Angew. Chem., Int. Ed., 2006, 45, 3748.

42 Y. Chauvin, Angew. Chem., Int. Ed., 2006, 45, 3741.

43 V. V. Ordomsky, A. Y. Khodakov, B. Legras and C. Lancelot, Catal. Sci. Technol., 2014, 4, 2896.

44 M. D’Auria, V. Frenna, M. Monari, A. P. Piccionello, R. Racioppi, D. Spinelli and L. Viggiani, Tetrahedron Lett., 2015, 56, 6598.

45 T. Miyazawa, T. Suzuki, Y. Kumagai, K. Takizawa, T. Kikuchi, S. Kato, A. Onoda, T. Hayashi, Y. Kamei, F. Kamiyama, M. Anada, M. Kojima, T. Yoshino and S. Matsunaga, Nat. Catal., 2020, 3, 851.

46 P. Wheeler, J. H. Phillips and R. L. Pederson, Org. Proc. Res. Dev., 2016, 20, 1182.

47 J. Kielhorn, C. Melber, D. Keller and I. Mangelsdorf, Int. J. Hyg. Environ. Health, 2002, 205, 417.

48 H. A. Schroeder and M. Mitchener, J. Nutr., 1971, 101, 1431.

49 I. Iavicoli, B. Bocca, L. Fontana, S. Caimi, A. Bergamaschi and A. Alimonti, Toxicol. Ind. Health, 2010, 26, 183.

50 J. Bunger, J. Stork and K. Stalder, Int. Arch. Occup. Environ. Health, 1996, 69, 33.

51 C. M. Bragadin, L. Ramani, L. Samer, G. Mestroni and G. Zassinovich, Antimicrob. Agents Chemother., 1975, 7, 825.

52 I. Iavicoli, V. Cufino, M. Corbi, M. Goracci, E. Caredda, A. Cittadini, A. Bergamaschi and A. Sgambato, Toxicol. In Vitro, 2012, 26, 963.

53 K. S. Andersen, Mutat. Res., 1979, 67, 209.

54 R. E. Yasbin, C. R. Matthews and M. J. Clarke, Chem.-Biol. Interact., 1980, 31, 355.

55 N. Vedeanu, C. Voica, D. A. Magdas, B. Kiss, M.-G. Stefan, R. Simedrea, C. Georgiu, C. Berce, O. Vostinaru, R. Boros, I. Fizesan, M. E. Rusu, A. Grozav, F. Loghin and D.-S. Popa, Environ. Chem., 2020, 17, 163.

56 J. T. P. Matshwele, F. Nareetsile, D. Mapolelo, P. Matshameko, M. Leteane, D. O. Nkwe and S. Odisitse, J. Chem., 2020, 2150419.

57 X. Jia, T. Wang, X. Bu, Q. Tu and S. Spencer, J. Pharm. Biomed., 2006, 41, 43.

58 Y. V. Yi and A. Masuda, Anal. Chem., 1996, 68, 1444.

59 P. Suresh, I. A. Azath and K. Pitchumani, Sens. Actuators, B, 2010, 146, 273.
60 T. Kangwanwong, W. Pluempanupat, W. Parasuk, H. E. Keenan and A. Songsasen, Sci. Asia, 2012, 38, 278.

61 B. Chen, F. Song, S. Sun, J. Fan and X. Peng, Chem. - Eur. J., 2013, 19, 10115.

62 S. Huang, F. Zhu, H. Qiu, Q. Xiao, Q. Zhou, W. Su and B. Hu, Colloids Surf., B, 2014, 117, 240.

63 N. Du, J. Song, S. Li, Y.-X. Chi, F.-Y. Bai and Y.-H. Xing, ACS Appl. Mater. Interfaces, 2016, 8, 28718.

64 V. Muthukumar and S. K. Iyer, Sens. Actuators, B, 2018, 267, 373.

65 C. Y. K. Chan, J. W. Y. Lam, C. K. W. Jim, H. H. Y. Sung, I. D. Williams and B. Z. Tang, Macromolecules, 2013, 46, 9494.

66 D. Maity, V. Kumar and T. Govindaraju, Org. Lett., 2012, 14, 6008 .

67 W. S. Hummers Jr. and R. E. Offeman, J. Am. Chem. Soc., 1958, 80, 1339.

68 Q. Zhao, C. Bai, W. Zhang, Y. Li, G. Zhang, F. Zhang and X. Fan, Ind. Eng. Chem. Res., 2014, 53, 4232.

69 L. Russo, F. Taraballi, C. Lupo, A. Poveda, J. J. Barbero, M. Sandri, A. Tampieri, F. Nicotra and L. Cipolla, Interface Focus, 2014, 4, 20130040.

70 G. Yasin, M. Arif, M. Shakeel, Y. Dun, Y. Zuo, W. Q. Khan, Y. Tang, A. Khan and M. Nadeem, Adv. Eng. Mater., 2018, 20, 1701166.

71 Q. T. Ain, S. H. Haq, A. Alshammari, M. A. A. Mutlaq and M. N. Anjum, Beilstein J. Nanotechnol., 2019, 10, 901.

72 A. Bhaumik, A. Haque, M. F. N. Taufique, P. Karnati, R. Patel, M. Nath and K. Ghosh, J. Material. Sci. Eng., 2017, 6, 1000364.

73 S. Indrawirawan, H. Sun, X. Duan and S. Wang, J. Mater. Chem. A, 2015, 3, 3432.

74 D. H. Wang, Y. Hu, J. J. Zhao, L. L. Zeng, X. M. Tao and W. Chen, J. Mater. Chem. A, 2014, 2, 17415.

75 D. Rosenthal, M. Ruta, R. Schlogl and L. K. Minsker, Carbon, 2010, 48, 1835.

76 A. Kaur, P. Chahal and T. Hogan, IEEE Electron Device Lett., 2016, 37, 142.

77 C. Y. Lung, M. G. Botelho, M. Heinonen and J. P. Matinlinna, Dent. Mater., 2012, 28, 863.

78 M. Kasha, Discuss. Faraday Soc., 1950, 9, 14-19.

79 J. B. Asha and P. Suresh, ACS Sustainable Chem. Eng., 2020, 8, 14301.

80 L. Jian, H.-Y. He, J. Huang, Q.-H. Wu, M.-L. Yuan, H.-Y. Fu, X.-L. Zheng, H. Chen and R.-X. Li, RSC Adv., 2017, 7, 23515. 81 A. Virgilio, A. B. S. Silva, A. R. A. Nogueira, J. A. Nóbrega and G. L. Donati, J. Anal. At. Spectrom., 2020, 35, 1614-1620.

82 H. Wang, J. Mao, A. Duan, B. Che, W. Wang, M. Ma and X. Wang, J. Fluoresc., 2013, 23, 323.

83 X. Sun, Y. Wang and Y. Lei, Chem. Soc. Rev., 2015, 44, 8019. 84 X. Li, S. Matthews and P. Kohli, J. Phys. Chem. B, 2008, 112, 13263. 